\title{
超高温复相陶瓷基复合材料烧蚀行为研究
}

\author{
琚印超 ${ }^{1,2}$, 刘小勇 ${ }^{2}$, 王 琴 $^{2}$, 张伟刚 ${ }^{3}$, 魏 殅 $^{2}$
}

(1. 北京航空航天大学 能源与动力工程学院, 北京 100191; 2. 中国航天科工集团公司北京动力机械研究所, 高 超声速冲压发动机技术重点实验室, 北京 $100074 ; 3$. 中国科学院 过程工程研究所, 多相复杂系统国家重点实验室, 北京 100190)

摘 要: 采用前驱体浸渍热解(PIP)工艺制备了 $\mathrm{ZrC}-\mathrm{SiC} 、 \mathrm{ZrB}_{2}-\mathrm{ZrC}-\mathrm{SiC}$ 和 $\mathrm{HfB}_{2}-\mathrm{HfC}-\mathrm{SiC}$ 复相陶瓷基复合材料, 复合 材料中的超高温陶瓷相均呈现出亚微米/纳米均匀弥散分布的特征, 对比研究了上述材料在大气等离子和高温电弧 风洞考核环境中的超高温烧蚀行为。研究结果表明, 超高温复相陶瓷基复合材料相比传统的未改性 $\mathrm{SiC}$ 基复合材料, 烧蚀后复合材料表面原位生成了固液两相致密氧化膜，两相协同作用实现了抗冲蚀和抗氧化的效果，对液相 $\mathrm{SiO}_{2}$ 的流失起到了阻碍作用，提升了材料的超高温烧蚀性能。在此基础上，提出了设计超高温复相陶瓷基复合材料应考 虑的因素。上述研究结果对陶瓷基复合材料在超高温有限寿命领域的应用具有一定的指导意义。

关 键 词: 超高温; 复相陶瓷基复合材料; 烧蚀行为; 基体设计; $\mathrm{SiC}$

中图分类号: V435 文献标志码: A

\section{Ablation Behavior of Ultra-high Temperature Composite Ceramic Matrix Composites}

\author{
JU Yinchao ${ }^{1,2}$, LIU Xiaoyong ${ }^{2}$, WANG Qin ${ }^{2}$, ZHANG Weigang $^{3}$, WEI Xi ${ }^{2}$
}

(1. School of Energy and Power Engineering, Beihang University, Beijing 100191, China; 2. Science and Technology on Scramjet Laboratory, Beijing Power Machinery Research Institute, China Aerospace Science and Industry Corporation Limited, Beijing 100074, China; 3. State Key Laboratory of Multiphase Complex Systems, Institute of Process Engineering, Chinese Academy of Sciences, Beijing 100190, China)

\begin{abstract}
Ultra-high temperature composite ceramic matrix composites $\mathrm{ZrC}-\mathrm{SiC}, \mathrm{ZrB}_{2}-\mathrm{ZrC}-\mathrm{SiC}$ and $\mathrm{HfB}_{2}-\mathrm{HfC}_{-} \mathrm{SiC}$ were fabricated by precursor infiltration and pyrolysis method. The ultra-high temperature ceramic phases in the materials were characterized by submicron/ nanometer uniform dispersion distribution. Ablation behaviors of $\mathrm{ZrC}-\mathrm{SiC}$, $\mathrm{ZrB}_{2}-\mathrm{ZrC}-\mathrm{SiC}$ and $\mathrm{HfB}_{2}-\mathrm{HfC}-\mathrm{SiC}$ matrix composites under atmospheric plasma and on-ground arc-jet wind tunnel were investigated comparatively. The main factors that affect design for ultra-high temperature composite ceramic matrix composites were summarized. The result shows that, compared with traditional SiC-based composites, ultra-high temperature composite ceramic matrix composites have a solid-liquid two-phase dense oxide film formed in situ on the surface of the composites after ablation. Synergistic effect of the two phases has achieved effects of erosion resistance and oxidation resistance, which plays a very important role in hindering the loss of liquid $\mathrm{SiO}_{2}$ and greatly improves the ultra-high temperature ablation performance of the materials. On this basis, the important factors that should be considered in the matrix design of ultra-high temperature composite ceramic matrix composites are obtained. The above results have instructional significance for the ultra-high temperature and the limited life application of
\end{abstract}

收稿日期：2021-03-23；收到修改稿日期：2021-05-21；网络出版日期：2021-06-01

作者简介：琚印超(1985-), 男，博士研究生. E-mail: by1704142@buaa.edu.cn JU Yinchao (1985-), male, PhD candidate. E-mail: by1704142@buaa.edu.cn

通信作者：魏 胥，高级工程师. E-mail: weixi31s@163.com WEI Xi, senior engineer. E-mail: weixi31s@163.com 
ceramic matrix composites.

Key words: ultra-high temperature; composite ceramics; matrix composites; ablation behavior; SiC

陶瓷基复合材料具有密度低、比强度高、热膨 胀系数小、耐高温、耐热冲击、耐烧蚀和假塑性断 裂等优异性能，在航空、航天等国防领域和新能源、 交通运输、高温化工等民用领域具有广阔的应用前 景 ${ }^{[1-3]}$ 。随着服役温度升高，基体分别采用玻璃基、 氧化物基和非氧化基复合材料。目前, 研究最多的 是以 $\mathrm{SiC}$ 为基体的复合材料, $\mathrm{SiC}$ 陶瓷基复合材料是 一种可在 $2000 \mathrm{~K}$ 以下温度的氧化气氛中长时间使 用的热结构材料, 但在更高温度及高速气流冲蚀的 服役环境中，例如高马赫数超燃冲压发动机燃烧室 环境, 单靠 $\mathrm{SiC}$ 基体无法保证复合材料具有优异的 综合服役性能, 通常需要引入超高温陶瓷相来提升 复合材料的超高温耐烧蚀性能 ${ }^{[4-5]}$ 。

超高温陶瓷通常是指 $\mathrm{Zr} 、 \mathrm{Hf} 、 \mathrm{Ta}$ 等过渡金属的 嗍化物、碳化物及氮化物，其中又以 $\mathrm{Zr} 、 \mathrm{Hf}$ 的嗍化 物和碳化物为主, 工程应用前景也最为广阔 ${ }^{[6-11]}$ 。在 $\mathrm{SiC}$ 基复合材料内部引入超高温陶瓷相的常规方法

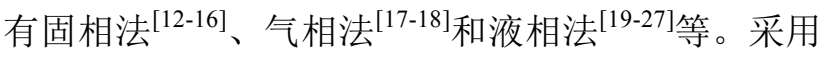
固相法引入的颗粒尺寸通常在 $1 \sim 10 \mu \mathrm{m}$, 并且分布 不均匀并伴有团聚现象, 因此超高温陶瓷相与 $\mathrm{SiC}$ 相之间的协同抗氧化耐烧蚀能力相对较弱。气相法 通常是采用化学气相渗透(CVI) 技术引入超高温陶 瓷相, $\mathrm{ZrCl}_{4} 、 \mathrm{CH}_{4}$ 以及 $\mathrm{BCl}_{3}$ 常作为锆源、碳源和硼 源，通过控制合适的沉积工艺和工装方式，可以气 相生长出 $\mathrm{ZrC}$ 或 $\mathrm{ZrB}_{2}$ 陶瓷。由于锆源的分子量大, 扩散系数比碳源和嗍源小, 因此 $\mathrm{ZrC}$ 或 $\mathrm{ZrB}_{2}$ 的 CVI 过程多发生在预制体的表面附近, 通常不易沉积到 厚壁构件的中心区域，而表面易出现封孔现象，所 以气相法沉积 $\mathrm{ZrC}$ 或 $\mathrm{ZrB}_{2}$ 通常用作表面的防氧化涂 层。另外, 用 CVI 法制备 $\mathrm{ZrB}_{2}(\mathrm{ZrC})-\mathrm{SiC}$ 复相陶瓷, 通常是先沉积一层 $\mathrm{ZrB}_{2} / \mathrm{ZrC}$, 再沉积一层 $\mathrm{SiC}$, 如 此反复多次从而达到复合的效果。由于超高温陶瓷 相与 $\mathrm{SiC}$ 相间的接触面积相对较小, 因此两相的协 同抗氧化耐烧蚀作用相对较弱。液相法通常采用反 应熔盐浸渗(RMI)的方法使熔融锆、铪合金与热解炭 反应生成 $\mathrm{ZrC}$, 或者通过浸渍热解工艺将不同含锆 有机前驱体引入到复合材料内部。含锆前驱体可分 为溶于乙醇等极性溶剂的前驱体或溶于甲苯等弱极 性溶剂的前驱体, 前者与最常用的 $\mathrm{SiC}$ 陶瓷有机前 驱体聚碳硅烷(Polycarbosilane, PCS)无法共溶于同 一种溶剂中, 只能将锆、铪前驱体与 PCS 分别浸渍 热解, 多次循环后制得 $\mathrm{ZrB}_{2}(\mathrm{ZrC})-\mathrm{SiC}$ 复合材料。因
此, $\mathrm{ZrB}_{2}(\mathrm{ZrC})$ 与 $\mathrm{SiC}$ 间的协同抗氧化烧蚀作用较弱, 后者可以与 PCS 溶于同一种溶剂, 因而可以实现 PCS 与超高温陶瓷前驱体的分子级共融熔。采用 PIP 法可以在一次循环过程中将超高温陶瓷相和 $\mathrm{SiC}$ 相 同时引入复合材料内部, 热解后可以得到亚微米甚 至纳米级均匀分布的复相陶瓷基体, 因此复相陶瓷 基体的协同耐烧蚀性能相对较好 ${ }^{[19-21,26-27] 。 ~}$

本研究以溶于弱极性溶剂的锆、铪有机前驱体 和聚碳硅烷的共混物为浸渍液, 采用前驱体浸渍热 解工艺分别制备了 $\mathrm{ZrC}-\mathrm{SiC} 、 \mathrm{ZrB}_{2}-\mathrm{ZrC}-\mathrm{SiC}$ 和 $\mathrm{HfB}_{2}-$ $\mathrm{HfC}-\mathrm{SiC}$ 复相陶瓷基复合材料。由于在 2000 2700 K 的超高温环境中使用时, 耐烧蚀性能是陶瓷基复合 材料最难以解决的核心问题, 因此, 本工作主要研 究了上述复合材料在大气等离子环境和电弧风洞环 境中的烧蚀行为, 从烧蚀性能方面考量, 提出了设 计超高温复相陶瓷基复合材料基体应考虑的重要因 素, 并给出了该领域后续研究的探索方向。

\section{1 实验方法}

\section{1 制备复合材料}

选用 T300 炭纤维二维针刺段为预制体(宜兴市 天鸟高新技术有限公司), 纤维体积分数为 $17 \%$ 。首 先采用等温 CVI 法在炭纤维表面沉积一层热解炭, CVI 工艺如下: 丙烷为碳源, 沉稳温度为 $1200 \mathrm{~K}$, 总压 $10 \mathrm{kPa}$, 气体流量 $4 \mathrm{~L} / \mathrm{min}$, 并控制停留时间在 $1 \mathrm{~s}$ 内。然后采用 PIP 工艺将 ZrC-SiC、 $\mathrm{ZrB}_{2}-\mathrm{ZrC}-\mathrm{SiC}$ 或 $\mathrm{HfB}_{2}-\mathrm{HfC}-\mathrm{SiC}$ 复相陶瓷基体分别引入复合材料 内部。采用中国科学院过程工程研究所研制的聚碳 锆氧烷、聚锆嗍烷、聚碳铪氧烷和聚铪硓烷作为 $\mathrm{ZrC} 、 \mathrm{ZrB}_{2} 、 \mathrm{HfC}$ 和 $\mathrm{HfB}_{2}$ 陶瓷有机前驱体 ${ }^{[28-29]}$, 采 用国防科技大学研制的聚碳硅烷为 $\mathrm{SiC}$ 有机前驱体, 将两类前驱体共混于二甲苯中形成 $\mathrm{ZrC}-\mathrm{SiC}$ 、 $\mathrm{ZrB}_{2}-\mathrm{ZrC}-\mathrm{SiC}$ 或 $\mathrm{HfB}_{2}$-HfC-SiC 复相陶瓷有机前驱 体。采用真空浸渍的方法, 通过毛细管力将不同的 复相陶瓷有机前驱体分别引入复合材料内的孔隙中, 而后在大气环境中 $400 \mathrm{~K}$ 交联和高温热解得到 $\mathrm{ZrC}-\mathrm{SiC} 、 \mathrm{ZrB}_{2}-\mathrm{ZrC}-\mathrm{SiC}$ 或 $\mathrm{HfB}_{2}-\mathrm{HfC}-\mathrm{SiC}$ 复相陶瓷 改性基体。热解工艺为氩气气氛, 热解温度 $1770 \mathrm{~K}$, 保温时间 $2 \mathrm{~h}$, 升温速率 $2 \mathrm{~K} / \mathrm{min}$ 。重复上述 PIP 过 程多次, 直到复合材料的开气孔率小于 $10 \%$ 。 


\section{2 表征与测试方法}

采用阿基米德排水法测试材料的密度及开孔 率。采用大气等离子和高温电弧风洞两种烧蚀环境, 在本实验室的高频等离子炬烧蚀试验机上进行大气 等离子烧蚀实验, 试样尺寸为 $30 \mathrm{~mm} \times 30 \mathrm{~mm} \times 10 \mathrm{~mm}$, 试验条件为电弧电压 $(65 \pm 5) \mathrm{V}$ 、电弧电流 $(440 \pm 10) \mathrm{A}$ 、 加热器功率约为 $30 \mathrm{~kW}$ 、氩气压力 $40 \mathrm{MPa}$ 、氩气流 量 $0.6 \mathrm{~L} / \mathrm{min}$ 、喷嘴直径 $8 \mathrm{~mm}$ 、烧蚀温度 $2450 \mathrm{~K}$; 采 用地面高温电弧风洞进行电弧风洞烧蚀试验, 试样 尺寸为 $100 \mathrm{~mm} \times 100 \mathrm{~mm} \times 5 \mathrm{~mm}$, 模拟来流条件为总 压 $0.6 \mathrm{MPa}$ 、温度 $2200 \sim 2300 \mathrm{~K}$ 、流速 0.6 马赫 $(1$ 马 赫 $=340 \mathrm{~m} / \mathrm{s}$ )、气氛为压缩空气。采用 $\mathrm{D} / \mathrm{max}-\mathrm{rB}$ 型 X 射线衍射仪和 JSM-5800 扫描电子显微镜(SEM)分 析表征烧蚀后材料的物相组成、显微组织和成分。

\section{2 结果与讨论}

\section{1 复合材料的微观结构}

\subsubsection{ZrC-SiC 复相陶瓷基复合材料}

图 1(a)为 $\mathrm{ZrC}-\mathrm{SiC}$ 复相陶瓷基复合材料的 XRD 图谱, 从图中可以看出, 经过高温热处理后, 有机 锆和聚碳硅烷完全反应转化为 $\mathrm{ZrC}$ 和 $\mathrm{SiC}^{[30]}$, 基体 的主要物相为 $\mathrm{ZrC} 、 \mathrm{SiC}$ 和 $\mathrm{C}$, 但由于炭纤维石墨化 程度较低, 衍射峰不显著。
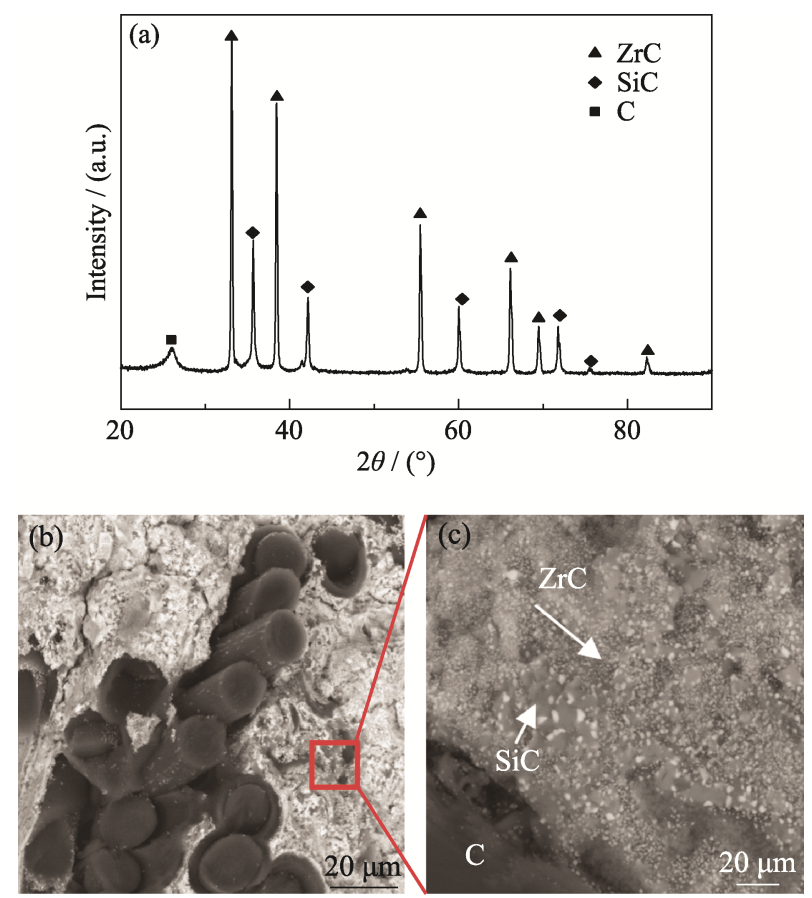

图 $1 \mathrm{ZrC}-\mathrm{SiC}$ 复相陶瓷基复合材料的 XRD 图谱(a)和 SEM 照片 $(\mathrm{b}, \mathrm{c})$

Fig. 1 XRD pattern (a) and SEM images (b, c) of ZrC-SiC matrix composites
图 1(b,c)为 $\mathrm{ZrC}-\mathrm{SiC}$ 复相陶瓷基复合材料的 SEM 照片, 图中黑色部分为炭纤维和热解炭基体, 灰色连续部分为 $\mathrm{SiC}$ 基体, 白色离散部分为 $\mathrm{ZrC}$ 基 体。 $\mathrm{ZrC}-\mathrm{SiC}$ 复相陶瓷基体呈现出纳米均匀弥散分 布的特征, $\mathrm{ZrC}$ 相的尺寸为 $50 \sim 200 \mathrm{~nm}$ 。一般认为, 超高温陶瓷相与 $\mathrm{SiC}$ 相的尺寸越小, 分散越均匀, 则复相陶瓷的耐烧蚀性能越好 ${ }^{[31]}$ 。纳米/亚微米 $\mathrm{ZrC}$ 颗粒均匀分散到 $\mathrm{SiC}$ 相中, 这种特有的微结构有利 于提高复合材料的抗氧化和耐烧蚀性能。

\subsection{2 $\mathrm{ZrB}_{2}-\mathrm{ZrC}-\mathrm{SiC}$ 复相陶瓷基复合材料}

图 2(a)为 $\mathrm{ZrB}_{2}-\mathrm{ZrC}-\mathrm{SiC}$ 复相陶瓷基复合材料的 $\mathrm{XRD}$ 图谱, 从图中可以看出含有六方相 $\mathrm{ZrB}_{2}$ 、立方 相 $\mathrm{ZrC}$ 、立方相 $\mathrm{SiC}$ 以及少量的 $\mathrm{C}$, 其中 $\mathrm{C}$ 是由复 合前驱体裂解生成的。经过高温热处理后形成了 $\mathrm{ZrB}_{2}-\mathrm{ZrC}-\mathrm{SiC}$ 复相陶瓷基复合材料。

图 2(b,c)为 $\mathrm{ZrB}_{2}-\mathrm{ZrC}-\mathrm{SiC}$ 复相陶瓷基复合材料 的 SEM 照片, 图中白色离散部分为 $\mathrm{ZrB}_{2}$ 和 $\mathrm{ZrC}$ 基 体, 尺寸为 $100 \sim 300 \mathrm{~nm}$, 呈现均匀弥散分布。

\subsection{3 $\mathrm{HfB}_{2}-\mathrm{HfC}-\mathrm{SiC}$ 复相陶瓷基复合材料}

图 3(a)为 $\mathrm{HfB}_{2}-\mathrm{HfC}-\mathrm{SiC}$ 复相陶瓷基复合材料的 $\mathrm{XRD}$ 图谱, 从图中可以看出经过高温热处理后, 基 体主要由 $\mathrm{HfB}_{2} 、 \mathrm{HfC} 、 \mathrm{SiC}$ 和 $\mathrm{C}$ 组成, 并含有微量 的 $\mathrm{HfO}_{2}$, 但由于前驱体自身的氧含量较高, 裂解过 程中碳化还原及嗍热还原不完全, 导致残留一定的 氧元素 ${ }^{[32]}$ 。
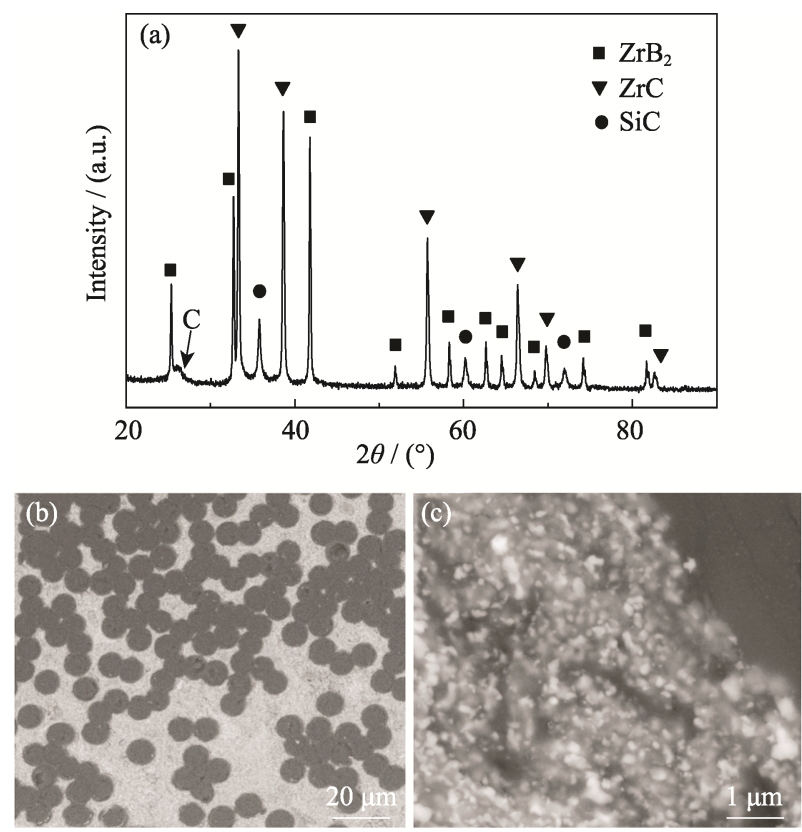

图 $2 \mathrm{ZrB}_{2}-\mathrm{ZrC}-\mathrm{SiC}$ 复相陶瓷基复合材料的 XRD 图谱(a)和 SEM 照片 $(b, c)$

Fig. 2 XRD pattern (a) and SEM images (b,c) of $\mathrm{ZrB}_{2}-\mathrm{ZrC}-\mathrm{SiC}$ matrix composites 

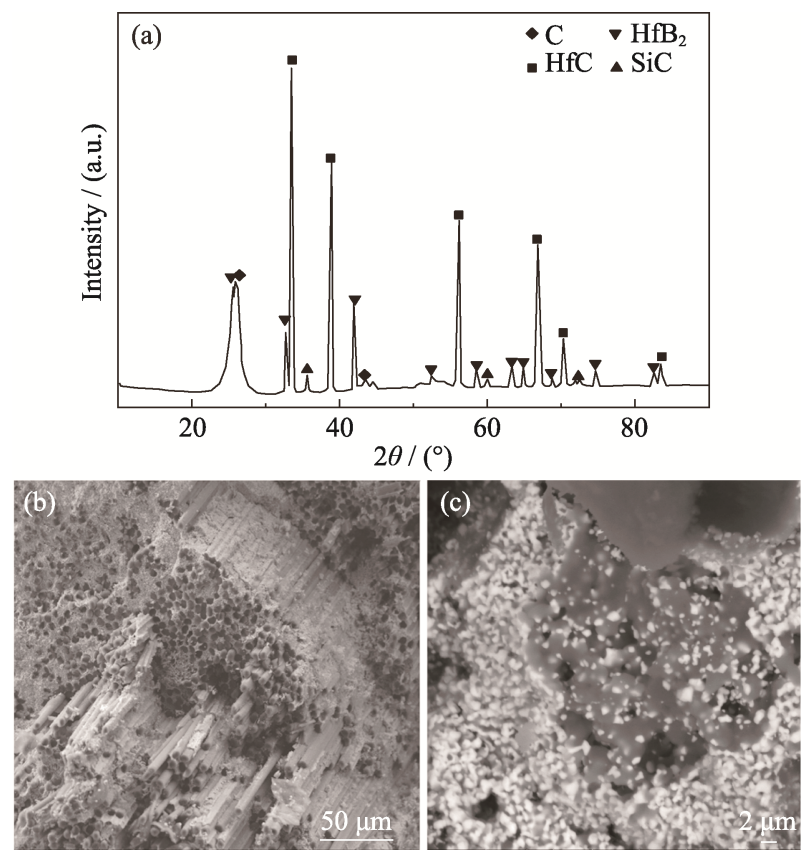

图 $3 \mathrm{HfB}_{2}-\mathrm{HfC}-\mathrm{SiC}$ 复相陶瓷基复合材料的 XRD 图谱(a)和 SEM 照片 (b, c)

Fig. 3 XRD pattern (a) and SEM images (b, c) of $\mathrm{HfB}_{2}-\mathrm{HfC}-\mathrm{SiC}$ matrix composites

图 3(b, c) 为 $\mathrm{HfB}_{2}-\mathrm{HfC}-\mathrm{SiC}$ 复相陶瓷基复合材料 的 SEM 照片, 白色离散部分为 $\mathrm{HfB}_{2}$ 和 $\mathrm{HfC}$ 基体，尺 寸为 $100 \sim 300 \mathrm{~nm}$, 呈均匀弥散分布状态。

\section{2 复合材料烧蚀实验结果对比}

\subsection{1 大气等离子烧蚀实验 $(2450 \mathrm{~K})$}

$\mathrm{ZrC}-\mathrm{SiC}$ 复相陶瓷基复合材料的线烧蚀率为 $0.59 \mu \mathrm{m} / \mathrm{s}$ ，远低于 $\mathrm{C} / \mathrm{SiC}$ 复相陶瓷基复合材料(对比 样)的线烧蚀率 $(15.97 \mu \mathrm{m} / \mathrm{s})$, 烧蚀后的表面微观形 貌如图 4(a,b)所示。从图 4(a,b)中可以看出, 复合材 料表面原位生成了一层致密的 $\mathrm{ZrO}_{2}-\mathrm{SiO}_{2}$ 复相氧化 物膜。在该烧蚀环境中, $\mathrm{ZrO}_{2}$ 为固相, $\mathrm{SiO}_{2}$ 为液相, 在协同作用下形成了既抗冲蚀(固相 $\mathrm{ZrO}_{2}$ 钉扎作用) 又抗氧化(液相 $\mathrm{SiO}_{2}$ 填充孔洞、弥合缺陷作用)的致 密氧化物保护层, 提升了复合材料的超高温耐烧蚀 性能。

$\mathrm{ZrB}_{2}-\mathrm{ZrC}-\mathrm{SiC}$ 复相陶瓷基复合材料线烧蚀率为 $1.32 \mu \mathrm{m} / \mathrm{s}$, 略高于 $\mathrm{ZrC}-\mathrm{SiC}$ 复相陶瓷基复合材料的 线烧蚀率，图 4(c, d) 为其烧蚀后的表面微观形貌。 从图 4(c, d)中可以看出, 复合材料表面原位生成了 一层结构完整的 $\mathrm{ZrO}_{2}-\mathrm{SiO}_{2}$ 复相氧化物膜, 在两相 分布均匀区域为致密氧化膜，而在富含 $\mathrm{SiO}_{2}$ 的区域 存在一些气孔, 这是由于 $\mathrm{ZrB}_{2}$ 氧化生成的 $\mathrm{B}_{2} \mathrm{O}_{3}$ 在 考核温度下的饱和蒸汽压高，气体挥发形成气孔， 同时破坏了复合材料表面生成的致密复相膜，导致 下层复合材料进一步发生氧化，因此在等离子烧蚀
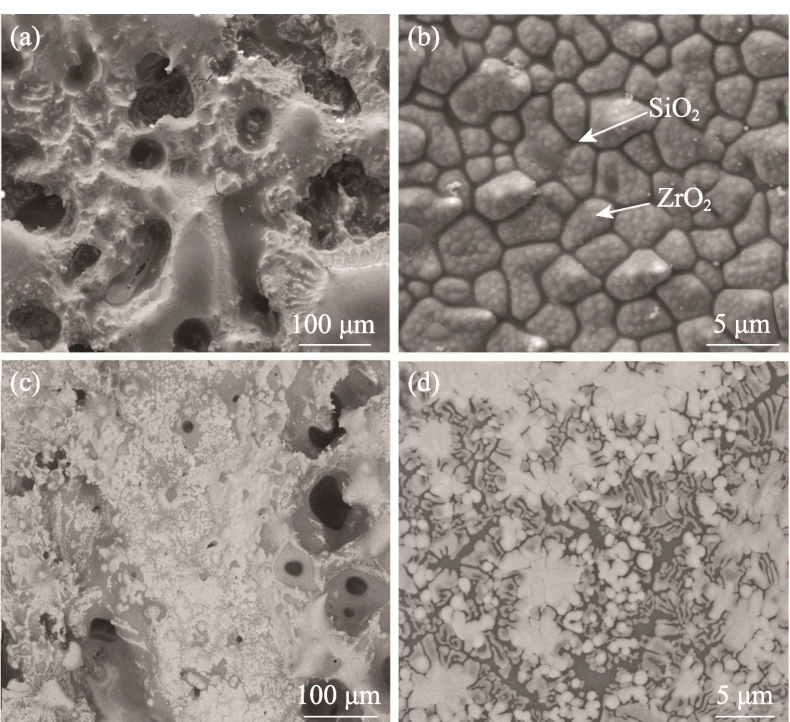

图 $4 \mathrm{ZrC}-\mathrm{SiC}(\mathrm{a}, \mathrm{b})$ 和 $\mathrm{ZrB}_{2}-\mathrm{ZrC}-\mathrm{SiC}(\mathrm{c}, \mathrm{d})$ 复相陶瓷基复合材 料经大气等离子烧蚀 $(2450 \mathrm{~K})$ 后的表面 SEM 照片

Fig. 4 SEM images of ablation centers of $\mathrm{ZrC}-\mathrm{SiC}(\mathrm{a}, \mathrm{b})$ and $\mathrm{ZrB}_{2}-\mathrm{ZrC}-\mathrm{SiC}(\mathrm{c}, \mathrm{d})$ matrix composites (ablated at $2450 \mathrm{~K}$ )

环境中 $\mathrm{ZrB}_{2}-\mathrm{ZrC}-\mathrm{SiC}$ 复相陶瓷基复合材料比 $\mathrm{ZrC}-\mathrm{SiC}$ 复相陶瓷基复合材料的抗烧蚀性能略差。

\subsection{2 高温电弧风洞烧蚀实验 $(2200 \mathrm{~K})$}

$\mathrm{ZrC}-\mathrm{SiC}$ 复相陶瓷基复合材料的抗烧蚀性能急 剧下降, $2200 \mathrm{~K}$ 时的线烧蚀率为 $9.8 \mu \mathrm{m} / \mathrm{s}$, 这是由于 表面复相氧化膜的完整性遭到了破坏。只有形成致 密的氧化膜才能阻止复合材料的进一步烧蚀, C 相 氧化在材料表面留下的孔洞以及原有的气孔要由陶 瓷相氧化的体积膨胀填充。在等离子烧蚀环境中, 由于等离子焰流垂直于试样表面, 烧蚀中心主体气 流流速慢, $\mathrm{SiO}_{2}$ 流失主要是由气相扩散传质到低流 速的主体气流中, 因而流失速率低, $\mathrm{ZrC}-\mathrm{SiC}$ 基体氧 化后生成的 $\mathrm{ZrO}_{2}-\mathrm{SiO}_{2}$ 复相陶瓷膜的完整性保持良 好; 而在电弧风洞环境中, 由于气流平行于材料表 面, 主体气流流速快, 对流传质对 $\mathrm{SiO}_{2}$ 流失起到主 导作用, $\mathrm{ZrC}$ 氧化后体积膨胀小, 很难完全填充 $\mathrm{C}$ 相 氧化后留下的孔洞, 逐渐造成复合材料表面氧化膜 中形成较大的贯穿性孔洞，导致 $\mathrm{SiO}_{2}$ 的流失，并成 为氧化性气氛的气相扩散通道直达下层复合材料表 面，下层氧化又导致表层氧化膜与复合材料间的黏附 力下降, 最终导致表层氧化膜因气流的机械冲刷而脱 落, 烧蚀率大幅增加。图 5 为 $\mathrm{ZrC}-\mathrm{SiC}$ 复相陶瓷电弧 风洞烧蚀后的表面形貌，材料表面原位生成了致密的 氧化膜，但氧化膜中存在大小不等的孔洞和裂纹。

$\mathrm{ZrB}_{2}-\mathrm{ZrC}-\mathrm{SiC}$ 复相陶瓷基复合材料的线烧蚀率 仅为 $0.33 \mu \mathrm{m} / \mathrm{s}$, 比 $\mathrm{ZrC}-\mathrm{SiC}$ 复合材料的抗烧蚀性能 大幅提升, 这是由于加入 $\mathrm{ZrB}_{2}$ 相增大了氧化后氧化 


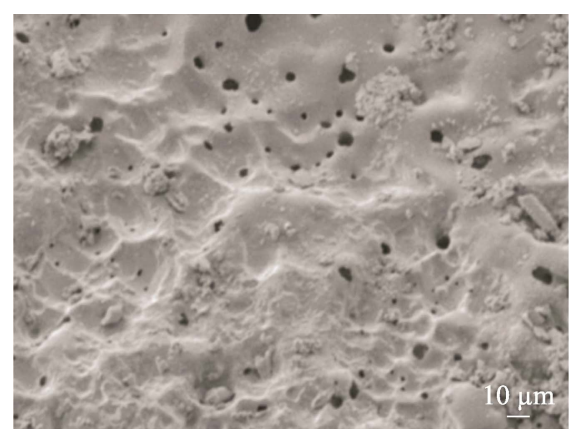

图 $5 \mathrm{ZrC}-\mathrm{SiC}$ 复相陶瓷基复合材料经电弧风洞烧蚀(2200 K) 后表面氧化膜的 SEM 照片

Fig. 5 SEM image of ablation center of $\mathrm{ZrC}-\mathrm{SiC}$ matrix composites (ablated at $2200 \mathrm{~K}$ )

膜的体积膨胀，复合材料表面生成的复相氧化膜更 容易填充 $\mathrm{C}$ 相氧化后残留的孔洞。虽然生成的 $\mathrm{B}_{2} \mathrm{O}_{3}$ 相会快速挥发, 但由于 $\mathrm{ZrO}_{2}$ 相在复合材料表面的分 布已经覆盖了 $\mathrm{C}$ 相残留的孔洞，氧化膜的整体完整 性相对较好, $\mathrm{ZrO}_{2}$ 的空间网络结构复杂, $\mathrm{SiO}_{2}$ 相挥发 要经历的气相扩散路径更曲折，抗烧蚀性能提升。

$\mathrm{ZrB}_{2}-\mathrm{ZrC}-\mathrm{SiC}$ 复相陶瓷基复合材料中 $\mathrm{SiO}_{2}$ 相的 挥发可用 $\mathrm{SiO}_{2}$ 在空间网络结构中的传质表示, 即可 用多孔介质中的传质连续方程式(1)表示 ${ }^{[33]}$ :

$$
\frac{\partial C}{\partial t}+\nabla \cdot\left(-D_{\text {eff }} \nabla C+C \boldsymbol{U}\right)=R
$$

式中, $C$ 为 $\mathrm{SiO}_{2}$ 的摩尔浓度 $\left(\mathrm{mol} / \mathrm{m}^{3}\right) ; D_{\text {eff }}$ 为 $\mathrm{SiO}_{2}$ 在 $\mathrm{ZrO}_{2}-\mathrm{SiO}_{2}$ 多孔网络中的有效扩散系数 $\left(\mathrm{m}^{2} / \mathrm{s}\right) ; \boldsymbol{U}$ 为 $\mathrm{SiO}_{2}$ 在预制体中的速度矢量 $(\mathrm{m} / \mathrm{s}) ; R$ 为 $\mathrm{SiO}_{2}$ 的反应 速率 $\left(\mathrm{mol} /\left(\mathrm{m}^{3} \cdot \mathrm{s}\right)\right)$, 表示单位时间内, 单位体积中生 成 $\mathrm{SiO}_{2}$ 的摩尔质量; $\nabla$.表示散度算符; $\nabla C$ 表示浓 度梯度 $\left(\mathrm{mol} / \mathrm{m}^{4}\right) ; t$ 为时间 $(\mathrm{s})$ 。

流体在多孔介质中的扩散与多孔介质的结构参 数密切相关,一般可由式(2)表示:

$$
D_{\text {eff }}=\frac{\varepsilon}{\tau} D
$$

式中, $\varepsilon$ 为氧化膜中 $\mathrm{ZrO}_{2}$ 的体积分数; $D$ 为 $\mathrm{SiO}_{2}$ 自 扩散系数 $\left(\mathrm{m}^{2} / \mathrm{s}\right) ; \tau$ 为曲折度因子, 是一个经验系数, 用来描述流体通过多孔介质扩散的路径长度。

由式(1)和式(2)可知, 加大扩散路径的曲折度 会降低 $\mathrm{SiO}_{2}$ 相的扩散系数, 从而降低其流失速率, 因此复杂的 $\mathrm{ZrO}_{2}$ 空间网络结构对 $\mathrm{SiO}_{2}$ 相的流失起 到限制作用。由以上分析可知, 在 $\mathrm{ZrC}-\mathrm{SiC}$ 复相陶 瓷基复合材料中引入 $\mathrm{ZrB}_{2}$ 相提升了抗高温电弧风 洞的烧蚀性能。

\subsection{3 高温电弧风洞烧蚀实验 $(2300 \mathrm{~K})$}

当高温电弧风洞的考核温度提升到 $2300 \mathrm{~K}$ 时, $\mathrm{ZrB}_{2}-\mathrm{ZrC}-\mathrm{SiC}$ 复相陶瓷基复合材料的耐烧蚀性能严
重下降，线烧蚀率由 $0.33 \mu \mathrm{m} / \mathrm{s}$ 大幅增加至 $5.3 \mu \mathrm{m} / \mathrm{s}$ 。 图 6 为 $2300 \mathrm{~K}$ 电弧风洞考核后的氧化膜表面形貌, $\mathrm{ZrO}_{2}$ 与 $\mathrm{SiO}_{2}$ 分相严重, 表明亚微米 $\mathrm{ZrB}_{2} / \mathrm{ZrC}$ 原位 生成的 $\mathrm{ZrO}_{2}$ 有很强的烧结活性，在电弧风洞中通过 对流传质带走挥发性物质的现象显著增强，气流促 使结合力弱的固态 $\mathrm{ZrO}_{2}$ 颗粒相互靠近, 也促进了 $\mathrm{ZrO}_{2}$ 固相颗粒的烧结, 导致 $\mathrm{ZrO}_{2}$ 的复杂空间网络 结构消失, $\mathrm{SiO}_{2}$ 相挥发严重, 在材料表面出现不同 尺度的气泡。 $\mathrm{ZrO}_{2}$ 相复杂空间网络结构消失及 $\mathrm{SiO}_{2}$ 加速流失导致材料表面的 $\mathrm{ZrO}_{2}-\mathrm{SiO}_{2}$ 复相保护膜失 效，最终材料烧蚀量大幅增加。

$\mathrm{HfB}_{2}-\mathrm{HfC}-\mathrm{SiC}$ 复相陶瓷基复合材料的线烧蚀率 仅为 $0.17 \mu \mathrm{m} / \mathrm{s}$ ，远低于相同条件下 $\mathrm{ZrB}_{2}-\mathrm{ZrC}-\mathrm{SiC}$ 复 相陶瓷基复合材料的线烧蚀率，耐烧蚀和抗氧化性 能提升显著，烧蚀后的表面复相氧化膜的微观形貌 见图 7, 表面原位生成的致密 $\mathrm{HfO}_{2}-\mathrm{SiO}_{2}$ 复相氧化膜

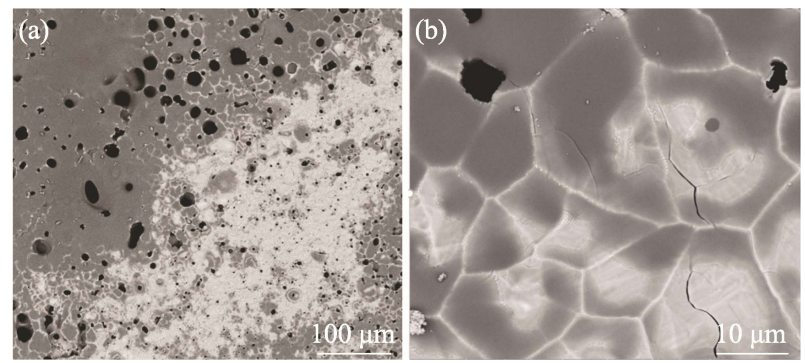

图 $6 \mathrm{ZrB}_{2}-\mathrm{ZrC}-\mathrm{SiC}$ 复相陶瓷基复合材料经电弧风洞烧蚀 $(2300 \mathrm{~K})$ 后表面氧化膜的 SEM 照片

Fig. 6 SEM images of ablation center of $\mathrm{ZrB}_{2}-\mathrm{ZrC}-\mathrm{SiC}$ matrix composites (ablated at $2300 \mathrm{~K}$ )
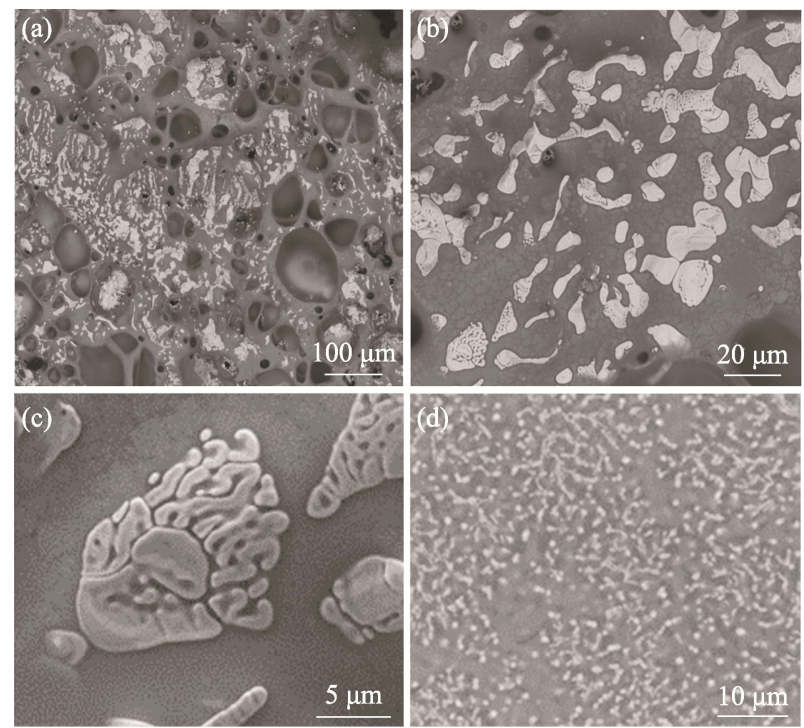

图 $7 \mathrm{HfB}_{2}-\mathrm{HfC}-\mathrm{SiC}$ 复相陶瓷基复合材料经电弧风洞烧蚀 $(2300 \mathrm{~K})$ 后表面氧化膜的 SEM 照片

Fig. 7 SEM images of ablation center of $\mathrm{HfB}_{2}-\mathrm{HfC}-\mathrm{SiC}$ matrix composites (ablated at $2300 \mathrm{~K}$ ) 
保存完好，存在少量气泡。 $\mathrm{HfO}_{2}$ 为固相, $\mathrm{SiO}_{2}$ 为液相。 对比图 6 和图 7, 在相同 $2300 \mathrm{~K}$ 电弧风洞考核 下, $\mathrm{HfO}_{2}$ 比 $\mathrm{ZrO}_{2}$ 的空间网络结构复杂。在 $\mathrm{HfO}_{2}-\mathrm{SiO}_{2}$ 复相氧化膜中, $\mathrm{HfO}_{2}$ 的空间网络结构涵盖着从几十 纳米至几十微米的多个尺度范围, 液相 $\mathrm{SiO}_{2}$ 填充 $\mathrm{HfO}_{2}$ 相间的孔洞, 复杂的空间网络结构阻碍了气相 $\mathrm{SiO}_{2}$ 的扩散，最终导致 $\mathrm{HfB}_{2}-\mathrm{HfC}-\mathrm{SiC}$ 复相陶瓷基复 合材料具有优异的抗电弧风洞烧蚀性能。同时，纳 米陶瓷颗粒的烧结活性受其熔点影响, 烧结温度越 靠近熔点, 其烧结活性越高。由于 $\mathrm{ZrO}_{2}$ 的熔点低于 $\mathrm{HfO}_{2}$, 导致在相同烧蚀条件下 $\mathrm{ZrO}_{2}$ 的烧结活性强 于 $\mathrm{HfO}_{2}$, 因此 $\mathrm{ZrO}_{2}$ 更易于烧结, 而 $\mathrm{HfO}_{2}$ 能更有效 地保持其复杂的空间网络结构, 最终使 $\mathrm{HfO}_{2}-\mathrm{SiO}_{2}$ 氧化膜的空间网络结构能够在更高的温度下保持整 体结构的完整性, $\mathrm{HfO}_{2}$ 对 $\mathrm{SiO}_{2}$ 的流失起到了钉扎和 阻碍作用。

表 1 汇总了实验结果, 可以看出材料的孔隙率 对烧蚀性能的影响相对较小, 在复合材料内部引入 复相陶瓷提升了超高温条件下的抗氧化耐烧蚀性能。

\section{3 设计超高温复相陶瓷基复合材料应考虑}

\section{的因素}

在各种不同测试环境和条件下，超高温陶瓷基 复合材料表面原位生成氧化膜的完整性是保障材料 具有优异烧蚀性能的基本条件; 而在表面氧化物膜 整体保持完整的前提下, 液态氧化物的流失速度是 决定表面氧化物膜完整性的核心因素之一; 而复合 材料表面原位生成固态氧化物空间网络结构的复杂 程度是决定液相氧化物流失难易程度的核心因素之 一。固相氧化物的空间结构越复杂, 则对 $\mathrm{SiO}_{2}$ 相流 失的制约作用越大，固液两相氧化膜能够在更长的 时间内保持结构完整性。超高温陶瓷的颗粒越细、 分布越均匀, 则生成的固相氧化物的结构越复杂; 而生成的固相氧化物熔点越高, 则同等条件下烧结 扩散的活性越低, 固相氧化膜便能在更高的温度下
保持空间网络结构的稳定性和复杂性，因而复合材 料的抗烧蚀性能越好。

基于以上结果，从烧蚀性能方面考量，设计超 高温复相陶瓷基复合材料应考虑以下因素：原位生 成氧化物膜的完整性; 液态氧化物的流失速度; 固 液两相复合氧化膜中固相空间网络结构的复杂性; 固相空间网络结构的稳定性。应继续开展如下进行 研究:

1)为了降低液态氧化物的流失速度，可在超高 温复相陶瓷基复合材料中加入熔点高于 $\mathrm{SiO}_{2}$ 且在 服役环境中呈玻璃态的物质，在更高的温度下起到 封孔作用。

2)为了提升固液两相复合氧化膜中固相空间网 络结构的复杂性，可进一步细化超高温陶瓷相的颗 粒，由目前的亚微米级均匀分布提升至纳米级均匀 分布，可提升复合材料本征超高温烧蚀性能。

3)为了提升固相氧化物空间网络结构的稳定性, 可引入 $\mathrm{HfO}_{2}$ 或 $\mathrm{ZrO}_{2}$ 烧结针化剂, 降低烧结活性, 使 其在更高的温度下保持原位生成的固相氧化物的空 间网络结构，提升复合材料的耐用温度。

\section{3 结论}

1) 采用 PIP 工艺制备了 $\mathrm{ZrC}-\mathrm{SiC} 、 \mathrm{ZrB}_{2}-\mathrm{ZrC}-\mathrm{SiC}$ 和 $\mathrm{HfB}_{2}-\mathrm{HfC}-\mathrm{SiC}$ 复相陶瓷基复合材料，复合材料中 的超高温陶瓷相呈现亚微米/纳米均匀弥散分布, 其 特征尺寸在 50 300 $\mathrm{nm}$; 复合材料的超高温烧蚀性 能优于传统的复合材料烧蚀性能。

2) $\mathrm{ZrC}-\mathrm{SiC}$ 复相陶瓷基复合材料在 $2450 \mathrm{~K}$ 大气 等离子环境中的线烧蚀率为 $0.59 \mu \mathrm{m} / \mathrm{s}$, 远低于 $\mathrm{C} / \mathrm{SiC}$ 复合材料对比样的线烧蚀率; 烧蚀后复合材 料表面原位生成了 $\mathrm{ZrO}_{2}-\mathrm{SiO}_{2}$ 固液两相致密氧化膜, 两相协同作用达到了既抗冲蚀又抗氧化的作用, 提 升了超高温烧蚀性能; 但在 $2200 \mathrm{~K}$ 电弧风洞实验中,

表 1 气孔率对不同材料烧蚀性能的影响

Table 1 Effect of porosity on ablation performance of different composites

\begin{tabular}{clccccc}
\hline Number & \multicolumn{1}{c}{ Sample } & $\begin{array}{c}\text { Density } \\
/\left(\mathrm{g} \cdot \mathrm{cm}^{-3}\right)\end{array}$ & $\begin{array}{c}\text { Porsity } \\
/ \%\end{array}$ & Experimental equipment & $\begin{array}{c}\text { Ablation } \\
\text { temperature } / \mathrm{K}\end{array}$ & $\begin{array}{c}\text { Linear ablation } \\
\text { rate } /\left(\mu \mathrm{m} \cdot \mathrm{s}^{-1}\right)\end{array}$ \\
\hline 1 & $\mathrm{C} / \mathrm{SiC}$ & 2.1 & 3.8 & atmospheric plasma torch & $2450 \mathrm{~K}$ & 15.97 \\
2 & $\mathrm{ZrC}-\mathrm{SiC}$ matrix composites & 2.2 & 16.3 & atmospheric plasma torch & $2450 \mathrm{~K}$ & 0.59 \\
3 & $\mathrm{ZrB}_{2}-\mathrm{ZrC}-\mathrm{SiC}$ matrix composites & 2.1 & 15.4 & atmospheric plasma torch & $2450 \mathrm{~K}$ & 1.32 \\
4 & $\mathrm{ZrC}-\mathrm{SiC}$ matrix composites $_{5}$ & 2.2 & 16.3 & art-jet wind tunnel testing & $2200 \mathrm{~K}$ & 9.8 \\
6 & $\mathrm{ZrB}_{2}$-ZrC-SiC matrix composites & 2.1 & 15.4 & art-jet wind tunnel testing & $2200 \mathrm{~K}$ & 0.33 \\
7 & $\mathrm{ZrB}_{2}-\mathrm{ZrC}-\mathrm{SiC}$ matrix composites & 2.1 & 15.4 & art-jet wind tunnel testing & $2300 \mathrm{~K}$ & 5.3 \\
\hline
\end{tabular}


表面氧化膜的整体结构不完整, 材料的耐烧蚀性能 明显下降, 线烧蚀率高达 $9.8 \mu \mathrm{m} / \mathrm{s}$ 。

3) $\mathrm{ZrB}_{2}-\mathrm{ZrC}-\mathrm{SiC}$ 复相陶瓷基复合材料在 $2450 \mathrm{~K}$ 大气等离子环境中的线烧蚀率为 $1.32 \mu \mathrm{m} / \mathrm{s}$, 在 $2200 \mathrm{~K}$ 电弧风洞中的线烧蚀率仅为 $0.33 \mu \mathrm{m} / \mathrm{s}$ 。在上 述两种考核环境中, 原位生成的 $\mathrm{ZrO}_{2}$ 相均能保持复 杂空间网络结构的完整性, 从而对 $\mathrm{SiO}_{2}$ 的流失起到 阻碍作用，表现出优异的耐烧蚀性能。

4) $\mathrm{ZrB}_{2}-\mathrm{ZrC}-\mathrm{SiC}$ 复相陶瓷基复合材料在 $2300 \mathrm{~K}$ 电弧风洞中的烧蚀性能下降严重, 线烧蚀率升至 $5.3 \mu \mathrm{m} / \mathrm{s}$, 而 $\mathrm{HfB}_{2}-\mathrm{HfC}-\mathrm{SiC}$ 复相陶瓷基复合材料在 该条件下依然具有极其优异的抗烧蚀性能, 线烧蚀 率仅为 $0.17 \mu \mathrm{m} / \mathrm{s}$ 。

\section{参考文献:}

[1] NASLAIN R. Design, preparation and properties of non-oxide $\mathrm{CMCs}$ for application in engines and nuclear reactors: an overview. Comp. Sci.\& Tech., 2004, 64(2): 155-170.

[2] 张立同, 成来飞, 徐永东. 新型碳化硅陶瓷基复合材料的研究 进展. 航空制造工艺, 2003, 1: 24-32.

[3] 张立同, 成来飞. 连续纤维增韧陶瓷基复合材料可持续发展战 略探讨. 复合材料学报, 2007, 2(24): 1-6.

[4] 段刘阳, 罗否, 王一光. 超高温陶瓷基复合材料的改性和烧蚀 行为. 中国材料进展, 2015, 34(10): 762-769.

[5] 董绍明, 周海军, 胡建宝, 等. 浅析极端环境下服役陶瓷基复合 材料的构建. 中国材料进展, 2015, 3(10): 742-750.

[6] OPEKA M, TALMY I, ZAYKOSKI J. Oxidation-based materials selection for $2000{ }^{\circ} \mathrm{C}$ plus hypersonic aerosurfaces: theoretical considerations and historical experience. J. Mater. Sci., 2004, 39(19): $5887-5904$.

[7] 张幸红, 胡平, 韩杰才, 等. 超高温陶瓷复合材料的研究进展. 科学通报, 2015, 3(60): 257-266.

[8] 张国军, 刘海涛, 邹冀, 等. 嗍化锆陶瓷生命周期中的化学反应. 科学通报, 2015, 60(3): 276-286.

[9] LESPADE P, RICHET N, GOURSAT P. Oxidation resistance of $\mathrm{HfB}_{2}-\mathrm{SiC}$ composites for protection of carbon-based materials. Acta Astronautica, 2007, 60(10/11): 858-864.

[10] MONTEVERDE F, BELLOSI A. Oxidation of $\mathrm{ZrB}_{2}$-Based ceramics in dry air. J. Electrochem. Soc., 2003, 150(11): B552-B559.

[11] OPILA E, HalbiG M. Oxidation of $\mathrm{ZrB}_{2}$-SiC. Ceram. Eng. Sci. Proc., 2001, 22(3): 221-228.

[12] TANG S F, DENG J Y, WANGSHIJUN, et al. Ablation behaviors of ultra-high temperature ceramic composites. Mater. Sci. \& Eng. A, 2007, 465(1/2): 1-7.

[13] WANG Y G, LIU W, CHENG L F, et al. Preparation and properties of $2 \mathrm{D} \mathrm{C} / \mathrm{ZrB}_{2}-\mathrm{SiC}$ ultra high temperature ceramic composites. Mater. Sci. \& Eng. A, 2009, 524:129-133.

[14] 童长青, 成来飞, 刘永胜, 等. $2 \mathrm{D} \mathrm{C} / \mathrm{SiC}-\mathrm{ZrB}_{2}$ 复合材料的烧蚀性
能. 航空材料学报, 2012, 32(2): 69-74.

[15] FENG Q, WANG Z, ZHOU H J, et al. Microstructure analysis of $\mathrm{C}_{\mathrm{f}} / \mathrm{SiC}-\mathrm{ZrC}$ composites in both fabrication and plasma wind tunnel testing processes. Ceram. Int., 2014, 40(1): 1199-1204.

[16] WANG Y, XU Y D, WANG Y G, et al. Effects of TaC addition on the ablation resistance of C/SiC. Mater. Lett., 2010, 64: 2068-2071.

[17] PATTERSON M, HE S, FEHRENBACHER L, et al. Advanced HfC-TaC oxidation resistant composite rocket thruster. Mater. Manuf. Proc., 1996, 11: 367-379.

[18] SAYIR A. Carbon fiber reinforced hafnium carbide composites. $J$. Mater Sci., 2004, 39: 5995-6003.

[19] ZHANG W G, XIE CH M, WEI X, et al. MAX Phase and Ultra-high Temperature Ceramics for Extreme Environments (Chapter $14 \mathrm{C} / \mathrm{C} \mathrm{ZrB} 2-\mathrm{ZrC}-\mathrm{SiC}$ Composites Derived from Polymeric Precursor Infiltration and Pyrolysis Part 2: Mechanical and Ablation Properties.) IGI Global, 2013: 435-460.

[20] ZHANG W G, XIE CH M, GE M, et al. MAX Phase and Ultra-high Temperature Ceramics for Extreme Environments (Chapter $13 \mathrm{C} / \mathrm{C}$ $\mathrm{ZrB}_{2}$-ZrC-SiC Composites Derived from Polymeric Precursor Infiltration and Pyrolysis Part 1: Preparation and microstructures.), IGI Global, 2013: 413-434.

[21] WEI X, ZHANG W G, GE M. Polymer-derived ZrC-SiC Composite Ceramic Powders. 39th International Conference on Advanced Ceramics and Composites, Daytona beach, USA, 2015. 01. 25-30.

[22] WANG Y G, ZHU X J, ZHANG L T, et al. C/C-SiC-ZrC composites fabrication reactive melt infiltration with $\mathrm{Si}_{0.87} \mathrm{Zr}_{0.13}$ alloy. Ceram. Int., 2012, 38(5): 4337-4343.

[23] WANG J, HU H F, ZHANG Y D, et al. Preparation and characterization of $\mathrm{C} / \mathrm{SiC}-\mathrm{ZrB}_{2}$ composites by precursor infiltration and pyrolysis. Ceram. Int., 2010, 36(3): 1011-1016.

[24] LI Q G, DONG S M, WANG Z, et al. Fabrication and properties of 3D $\mathrm{C}_{\mathrm{f}} / \mathrm{ZrC}-\mathrm{SiC}$ composites using $\mathrm{ZrC}$ precursor and polycarbosilane. J. Am. Ceram. Soc., 2012, 38(7): 6041-6045.

[25] YAN CH L, LIUN R J, CAO Y B, et al. Preparation and properties of 3D needle-punched C/ZrC-SiC composites by polymer infiltration and pyrolysis process. Ceram. Int., 2014, (40): 10961-10970.

[26] 武海棠. 易加工超高温陶瓷复合材料的制备与性能研究. 北京: 中国科学院过程工程研究所博士学位论文, 2011.

[27] 谢昌明. 整体抗氧化超高温复合材料研究. 北京: 中国科学院 过程工程研究所硕士学位论文, 2012.

[28] 张伟刚, 戈敏, 魏笚. 一种碳化锆和二嗍化锆陶瓷有机前驱体 材料及其制备方法. 国防发明专利: 201110010110.2.

[29] 张伟刚, 戈敏, 魏笚. 一种碳化铪和二嗍化铪陶瓷有机前驱体 材料及其制备方法. 国防发明专利: 201218002692.5 .

[30] HU P, WANG G, WANG Z. Oxidation mechanism and resistance of $\mathrm{ZrB}_{2}-\mathrm{SiC}$ composites. Corrosion Sci., 2009, 51: 2724-2732.

[31] WU H T, WEI X, YU SH Q, et al. Ablation performances of multi-phased $\mathrm{C} / \mathrm{C}-\mathrm{ZrC}-\mathrm{SiC}$ ultra-high temperature composites. Journal of Inorganic Materials, 2011, 26(8): 852-856.

[32] 魏等, 李捷文, 张伟刚. $\mathrm{HfB}_{2}-\mathrm{HfC}-\mathrm{SiC}$ 改性 $\mathrm{C} / \mathrm{C}$ 复合材料的超高 温烧蚀性能研究. 装备环境工程, 2016, 13(3): 12-17.

[33] BIRD B, STEWART W, LIGHTFOOT E. Transport Phenomena. New York: John Wiley \& Sons, 1960. 